\title{
VERDAD Y JUICIO REFLEXIONANTE EN KANT
}

ISABEL CABRERA

INSTITUTO DE INVESTIGACIONES FILOSÓFICAS Universidad Nacional Autónoma de MÉxico

En el primer capítulo de su libro Mind and World, John Mc Dowell dibuja con especial agudeza el columpio en el que oscila frecuentemente la filosofía cuando pretende dar cuenta de las problemáticas relaciones entre la mente y el mundo. Por un lado, existe una tendencia hacia el coherentismo, que considera que sólo las creencias pueden ser fundamento de las creencias; por otro, hay la tendencia a acentuar la presencia de algo dado que constituye, en primera o en última instancia, el fundamento de nuestros conocimientos. El columpio se convierte en un dilema en tanto cada uno de los cabos implican dificultades insuperables: el coherentismo, que concibe el conocimiento como un sistema de creencias, parece despegarlo de aquella realidad que debería constituir su referencia, y no se ve claro cómo es que habría de restablecerse esta relación; la otra tendencia, por su parte, conlleva el problema de explicar en qué sentido lo dado puede convertirse en argumento de verdad: quienes apelan a este mito - dice Mc Dowellparecen ofrecer excusas más que razones, porque señalar la presencia de lo dado es señalar la presencia de un límite y no de un fundamento [Mc Dowell, 1994, p. 8].

Me parece que frente al problema de la verdad, la filosofía de Kant oscila en este columpio. En la Crítica de la razón pura, Kant hace explícita su adhesión a "la definición nominal de verdad, a saber, la conformidad del conocimiento con su objeto" [A 58/B 82] y se pregunta por un criterio general de verdad. La definición no puede, ella misma, constituir un criterio por razones obvias: el criterio exige una generalidad que nos obliga a prescindir del contenido específico de los juicios, sacrificando de esta manera su verdad - la cual precisamente consiste en el acuerdo entre dicho contenido específico y ciertos hechos del mundo [Cfr. A 58-59/B 83]. Pero entonces, ¿cuál es el criterio que nos permite reconocer y predicar verdad?, ¿cómo saber cuándo nuestros conocimientos coinciden con sus objetos? 
Al enfrentar esta pregunta, la filosofía de Kant comienza a balancearse en el columpio mencionado: por una parte, Kant a veces se refugia en un idealismo dentro del cual la verdad se reconoce por la coherencia orgánica de los conocimientos, por su conformidad a ciertos principios, por su sistematicidad; otras veces, en cambio, Kant se refugia en la patencia de algo dado, que no es anticipado por la mente sino producido por una realidad independiente; una mítica "cosa en si" parece ser la responsable última del contenido de nuestros conocimientos. En lo que sigue me detengo brevemente en las razones que encuentro en Kant para apoyar cada una de estas tendencias, para proponer después una vía intermedia de solución apelando a los principios del juicio reflexionante como criterios adicionales de verdad.

La tendencia de la filosofía de Kant al Idealismo, y con ella su tendencia al coherentismo, es innegable; no está de más, sin embargo, puntualizar algunas tesis que son precisamente las que hacen que esta tendencia se mueva en sentido opuesto, generando un columpio que a veces parece convertirse en dilema. En su Jäsche Logic, Kant señala un problema de la definición nominal de verdad, cuya mención omite en la Crítica. Dado que la verdad es una relación de conformidad entre el conocimiento y su objeto, la cuestión es cómo acceder a los objetos para decidir si se conforman o no con los supuestos conocimientos. Parece innegable que nuestro acceso a la realidad es siempre a través de las representaciones que nos hacemos de ella, de tal manera que juzgar acerca del valor de verdad de un juicio nos lleva, irremediablemente, a otros juicios. La situación en la que estamos es similar - dice Kant - a la que enfrenta un jurado cuando debe decidir sobre la responsabilidad de un hombre que se declara inocente, apelando a otro testigo que asegura que el culpado no miente, y así sucesivamente [Kant, 1800, Int. § VII]; no hay nada sino otros juicios, otros conocimientos, $\mathrm{u}$ otros testimonios, en los cuales basar nuestras pretensiones de verdad. Juzgar sobre la conformidad del conocimiento con su objeto nos lleva a confrontar un tipo de juicios con otro tipo de juicios.

La dificultad de acceder directamente a los objetos, sin mediación de las representaciones, constituye en este contexto un excelente argumento en favor del idealismo. La verdad parece convertirse en una relación, no entre dos entidades diferentes: proposiciones y hechos, sino entre dos distintos tipos de representaciones: conceptos e intuiciones. Pero si la verdad es en última instancia una relación entre representaciones, entonces la coherencia y la sistematicidad adquieren vital importancia. De aquí que Kant insista una y otra vez, sobre todo al final de su primera Crítica, en que el conocimiento no es un mero agregado de representaciones y juicios sino un sistema que 
posee organicidad [Cfr. A 642 ss./B 670 ss. y A 832 ss./B 860 ss.]. Pero vayamos más despacio.

La búsqueda que emprende Kant de un criterio de verdad lo lleva siempre a principios. En las Lógicas, Kant está preocupado por leyes y principios muy generales, que constituyen condiciones negativas de cualquier conocimiento: el principio de no contradicción, el principio de razón suficiente y la ley del tercero excluido; mientras que la Crítica se ocupa más bien de los principios que constituyen la lógica trascendental, es decir de aquellos principios que se derivan de las categorías o conceptos a priori de la experiencia, y la condicionan de manera fundamental. Son estos principios de la experiencia aquello que permite trazar la distinción básica entre experiencia objetiva y subjetiva, son estas categorías las responsables, de acuerdo con algunos pasajes de la Crítica, de la unidad que presenta el objeto [Cfr. A 108 ss.] y, por consiguiente, del rasgo que hace posible considerarlo como referente del conocimiento [Cfr. A 191/B 236].

No obstante, aunque dichos criterios son suficientes para la objetividad, no son suficientes para la verdad. ${ }^{1}$ Categorías y principios no bastan para producir conocimiento verdadero; de hecho, cuando lo intentan conducen a la razón humana a alguna de sus ilusiones dialécticas. Para alcanzar verdad, los principios de la lógica trascendental han de relacionarse con algo más que ideas. De aquí que la lógica trascendental, como la lógica formal, sea sólo un conjunto de criterios negativos de verdad, es decir, necesarios pero no suficientes para la verdad empírica.

Pero, ¿en qué sentido dichos principios funcionan como criterios de verdad? Los principios de la lógica formal son criterios negativos de verdad porque nos permiten excluir de entrada aquellos conocimientos que no están "de acuerdo consigo mismos". Los principios de la lógica trascendental son, por su parte, criterios negativos de verdad porque determinan el ámbito de los objetos que pueden sernos dados, hacen explícitas las condiciones generales que deberá cumplir cualquier juicio para considerarse objetivo, es decir, para ser considerado como candidato a la verdad. Veamos un ejemplo: que el principio de causalidad sea parte de la lógica trascendental, y por consiguiente, sea un criterio negativo de verdad, significa que, cuando observamos un fenómeno: el calentamiento de una piedra, para seguir un ejemplo del propio Kant, debemos buscar causas espacio temporales observables, o relacionadas con lo observable, que expliquen dicho fenómeno de acuerdo con una regla. El principio abre un ámbito de posibilidades que, sin embargo, queda indeterminado. Para elegir entre las causas posibles no basta la categoría de causalidad y su principio asociado, hay que apelar

1 Con respecto a las verdades analíticas, los principios lógicos son, además de necesarios, suficientes. No obstante ningún principio es suficiente para la verdad sintética. 
además a las experiencias específicas. Son ellas las que nos darán las razones concretas, en cada caso, para preferir una explicación entre otras y decidir, por ejemplo, que fue el sol, y no el fuego, lo que calentó la piedra. De aquí que aunque el principio de causalidad sea para Kant sintético a priori, los juicios causales particulares son siempre proposiciones contingentes y, por consiguiente, corregibles [Cfr. A 766/B 974].

Así entonces, el acuerdo con principios no basta para reconocer las verdades empíricas más comunes. Estamos frente a las dificultades que hacen a Kant moverse hacia el otro extremo del columpio. Los principios lógicos garantizan verdades lógicas, los principios trascendentales dan condiciones de la experiencia posible, pero ¿qué hay respecto a las verdades empíricas, que ni la lógica ni los principios a priori bastan para garantizar?, ¿cuál es su criterio de verdad?

Ralph C.S. Walker dice que el Idealismo trascendental de Kant es un coherentismo incompleto porque hay un conjunto de proposiciones para las cuales la verdad no es una cuestión de coherencia: aquellos juicios que reportan lo que nos es dado en la percepción no son verdaderos en virtud de sus relaciones con otros juicios, sino en virtud de su correspondencia con hechos independientes de nuestras creencias. Esta indispensable referencia nos remite a las "cosas en si". De acuerdo con la interpretación de Walker, para Kant la verdad es una cuestión de coherencia entre las "condiciones formales de la experiencia" y su "condición material", es decir, la sensación, que en última instancia no es sino un conocimiento parcial de la realidad independiente [Walker, 1989, pp. 63-64]. De acuerdo con esta lectura, la realidad independiente es un complemento indispensable del criterio kantiano de verdad porque sólo ella puede respaldar nuestra creencia de que los objetos de conocimiento son públicos y afectan de manera similar a quienes los perciben [Walker, 1989, p. 77]. Lo dado pretende dejar de ser un misterio insondable para convertirse en un ámbito compartido al cual podemos referirnos intersubjetivamente.

A pesar de este esfuerzo por desmitificar la realidad en sí, creo que la interpretación de Walker ilustra, más bien que supera, la dificultad señalada por Mc Dowell. El que los fenómenos sean objetos públicos no convierte a lo dado en un argumento de verdad, más bien sugiere un recurso adicional para determinar la verdad de nuestros juicios de percepción: el acuerdo intersubjetivo. Pero si esto es así, el resultado nos devuelve al coherentismo: mis juicios de percepción son verdaderos, no si coinciden con hechos independientes, sino si coinciden con los juicios de percepción de otros sujetos. No logramos salir del círculo, la verdad es otra vez una cuestión de coherencia, de armonía entre proposiciones. 
Antes que Walker, Juan Miguel Palacios ofrece una lectura similar respecto al problema de la verdad en Kant. Palacios reconoce que tanto las verdades analíticas como las verdades trascendentales pueden establecerse por referencia a los principios de la lógica clásica y de la lógica trascendental kantiana. No obstante, refiere el criterio de verdad empírica al "segundo postulado del pensar empírico en general", aquel que conecta el concepto de realidad [Wirklichkeit] con la sensación [Palacios, 1979, pp. 135-148]. Lo dado es nuevamente el presupuesto que le permite a Kant salir del círculo originalmente señalado: a través de las sensaciones, las representaciones adquieren un ancla en la realidad [Cfr. A 251-252]. Sin embargo, otra vez, no es claro cómo esta ancla puede convertirse en un fundamento de conocimiento. Señalar que hay juicios que se basan en las sensaciones, las cuales son el efecto de nuestro contacto con la realidad, no resuelve la dificultad señalada, ya que ella consiste en exigir una explicación acerca de cómo esta supuesta causa (la realidad), y el efecto sensorial que produce en nosotros (la sensación), pueden convertirse en razones de verdad. Apelar a lo dado no deja de ser un gesto ostensivo, el señalamiento un límite, de algo que debe ser tomado en cuenta si no queremos confundir conocimiento con pensamiento coherente, pero está lejos de ser claro cómo habremos de integrar este elemento causal al "espacio de las razones" - para usar la expresión que Mc Dowell recoge de Sellars.

Pero cabe sospechar que Kant está consciente no sólo de la insuficiencia del coherentismo, sino también de las dificultades que señala Mc Dowell respecto al mito de lo dado. Es cierto que en la "Estética trascendental" Kant se refiere a las cosas en sí como aquellas entidades no espacio temporales que, sin embargo, nos afectan a través de la sensación y son la referencia última de nuestras representaciones. No obstante, más adelante en la Crítica, Kant parece corregir este sentido positivo del concepto de "cosa en sí", y habla del "noúmeno" como un concepto meramente negativo que no hace sino señalar un límite infranqueable [A 252/B 309]. Si tomamos en serio esta restricción, entonces parece claro que la conformidad que se da entre el conocimiento y su objeto no es la correspondencia con una realidad completamente independiente. Se trata más bien de una adecuación empírica, de una conformidad entre el conocimiento y sus fenómenos.

De acuerdo con Kant, todo juicio contingente se justifica en la sensación. Pero para ser objeto de un juicio, la sensación deberá adquirir el carácter espacio temporal, es decir, deberá convertirse en intuición. Ahora bien, estas intuiciones o representaciones singulares no afirman ni niegan nada, son "ciegas" sin conceptos; no constituyen, pues, ninguna plataforma de verdades básicas. En todo caso, lo que constituye una plataforma de verdades básicas son los juicios que elaboramos a partir de estas intuiciones, dentro 
de los cuales el más elemental parece ser aquel que conecta una intuición con un concepto empírico. Pero otra vez, ¿cómo saber cuándo este tipo de juicios son verdaderos? Las intuiciones (que no son sino sensaciones ubicadas espacio temporalmente) parecen imponer, por lo que respecta a su materia, ciertas restricciones, ya que no pueden ser conceptualizadas de cualquier manera. No obstante, la intuición no determina por completo el concepto que la describe. Lo dado en la sensación constituye un límite, una causa última, y hay un margen de indeterminación respecto a cómo conceptualizar dicha causa para integrarla al así llamado "espacio de las razones".

Estamos, me parece, en posición de señalar en qué sentido el juicio reflexionante podría servir para mitigar esta oscilación de la filosofía de Kant. Para ello, es importante recordar dos de las funciones que Kant le asigna en su Crítica del juicio: por un lado, el juicio reflexionante es responsable de buscar conceptos mediatos, es decir, de ofrecer conceptos empíricos para las intuiciones dadas, proceso central para la adecuación empírica; por otro lado, es responsable de la sistematicidad, complemento indispensable de la coherencia. Pero veamós con un poco más de detalle en qué sentido cada una de estas funciones ayudaría a solucionar el problema de la verdad empírica.

En la primera versión de su introducción a la Crítica del juicio, Kant dice que un principio básico de la reflexión es presuponer que "para todas las cosas naturales pueden hallarse conceptos empíricamente determinados"; de no presuponer esto, "todo reflexionar se llevaría a cabo a la ventura y ciegamente, $y$, por lo tanto, sin una fundada expectativa de su concordancia con la naturaleza" [Kant, 1790, 1a. Int. $\S \mathrm{v}$ ]. La introducción publicada, por su parte, dice que los juicios reflexionantes buscan un universal para un particular dado [Kant, 1790, A xxiv/B xxvi]. Lo que nos es dado a través de la sensación es conceptualizable, podemos darle un nombre para integrarlo al así llamado "espacio de las razones". El juicio reflexionante se ocupa, precisamente, de esta labor de identificar intuiciones dadas usando conceptos mediatos. Pero si el juicio reflexionante es el encargado de buscar conceptos empíricos para identificar intuiciones, entonces parece jugar un papel fundamental en la formulación de los juicios que hemos considerado más básicos: los reportes de percepción, aquellos juicios que conectan una intuición con un concepto empírico.

Es cierto que, una vez encontrado el concepto, los juicios subsiguientes serán determinantes, pero esto no impide que los juicios reflexionantes jueguen un papel decisivo en la producción misma de conceptos. El jui- 
cio reflexionante opera, básicamente, comparando singulares y acentuando similitudes y diferencias entre ellos. Muy probablemente, para encontrar conceptos mediatos sea indispensable recurrir a la inducción o la analogía, las cuales parecen ser dos formas de razonamiento que ejemplifican esta búsqueda de conceptos para identificar particulares dados. Lo importante será que sin juicios reflexionantes nunca podríamos identificar lo particular ni acceder de allí a otros niveles de generalidad, conformando paulatinamente un sistema de conocimiento. Al intentar una primera conceptualización de un particular dado, el juicio reflexionante integra los fenómenos en un sistema de experiencia y convierte las percepciones dadas en una experiencia coherente [Kant, 1790, A xxxii-xxxiii/B xxxiv-xxxv].

Sin embargo, es claro que no desaparece todo margen de indeterminación cuando conceptualizamos las intuiciones. Al constituir un límite, lo dado nos dice que no puede ser conceptualizado de cualquier manera, pero no trae consigo una etiqueta que lo identifique, no nos dice cuál es la mejor forma de conceptualizarlo. El juicio reflexionante es, así, indeterminado y corregible. No obstante, la búsqueda de sistematicidad que Kant también confiere a este juicio, permite reducir estos márgenes de indeterminación: para incluir lo dado en el ámbito de las razones, buscaremos el concepto que maximice la sistematicidad de nuestro conocimiento.

En ambas introducciones a su tercera Crítica, Kant insiste en señalar como presupuesto de nuestra facultad de juzgar la creencia de que la naturaleza es clasificable y sistematizable; que a pesar de su enorme diversidad, se deja clasificar a través de un sistema de principios y leyes, como si fuera afín a nuestra facultad de juzgarla [Kant, 1790, 1a. Int. $\S$ iv y A xxxv/B xxxvi]. El juicio reflexionante es el responsable de esta sistematización. En repetidas ocasiones, se ha señalado que no es claro qué es lo que añade esta función del juicio reflexionante a la labor que Kant otorga a las ideas regulativas al final de su primera Crítica [Cfr. A 642-668/B 670-696]. Lo que ocurre, en mi opinión, es que el juicio reflexionante complementa de manera indispensable la labor de sistematización de las ideas. Veamos brevemente por qué. $^{2}$

Las ideas son, para Kant, conceptos inevitables de la razón que, sin embargo, no refieren a ningún objeto. De aquí que tengan una función meramente regulativa; su labor es orientar el entendimiento hacia la búsqueda de dos ideales: la sistematicidad y la completud del conocimiento:

2 Para más detalle sobre las relaciones entre juicio reflexivo e ideas regulativas, véanse los textos de P. Guyer [1979, pp. 43-47; 1991, pp. 34-36 y 1996, supra, pp. 1-59]. Sea redundante o no, lo importante es que la función sistemática del juicio reflexivo lo convierte en herramienta de la coherencia 
el uso hipotético de la razón tiende a la unidad sistemática del conocimiento del entendimiento y esta unidad es el criterio de verdad de las reglas. [A 647/B 675]

Para lograr este ideal - siempre inalcanzable - las ideas regulativas echan mano de algunos criterios subsecuentes, como la economía de principios, la diversidad de consecuencias o especificaciones, y la continuidad o parentesco entre géneros y especies, a manera de ciertas virtudes epistémicas que hacen preferibles ciertas conceptualizaciones sobre otras cuando describimos fenómenos. Dice Kant:

La razón prepara, pues, el terreno del entendimiento de la manera siguiente:

1) mediante el principio de homogeneidad de lo diverso bajo géneros más elevados;

2) mediante un principio de variedad de lo homogéneo bajo especies inferiores; [y] para completar la unidad sistemática, la razón añade todavía,

3) la ley de afinidad de todos los conceptos; esta ley obliga siempre a pasar de una especie a otra sin romper la continuidad, incrementando gradualmente la variedad. Podemos llamarlos el principio de homegeneidad, de especificación y de continuidad de las formas. [A 657-658/B 685-686]

Así entonces, por mor de la sistematicidad, preferimos clasificar la diversidad dada, bajo un mínimo de principios que ofrezca, no obstante, una red suficientemente fina de especificaciones, capaz de reflejar la gama de diferencias graduales que presenta la diversidad natural. De acuerdo con esta sección de la primera Crítica, las ideas parecen capaces de realizar la labor de clasificación y sistematicidad que Kant otorgará en su última Crítica al juicio reflexionante.

No obstante, estos párrafos son explícitos en un punto crucial: la razón no produce conceptos "sino que simplemente los ordena y les da aquella unidad que pueden tener al ser ampliados al máximo" [A 643/B 671]. Es decir, las ideas no pueden ofrecer la gama de conceptos que median entre el principio más general y la última de sus especificaciones; lo que hacen más bien es ordenar de manera sistemática los conceptos empíricos dados. En este sentido, el juicio reflexionante resulta indispensable para complementar esta labor de las ideas, ya que se encarga de comparar y emparentar instancias singulares bajo conceptos mediatos, ubicándolas en una red conceptual lo suficientemente fina y económica como para tender a la unidad sistemática hacia la que nos orientan las ideas regulativas. De aquí que el juicio reflexionante sea una herramienta indispensable del criterio coherentista de verdad. 
Así pues, el juicio reflexionante es responsable de dos funciones que resultan complementos indispensables de los criterios de verdad reconocidos por Kant: la coherencia y la adecuación empírica; y en este sentido puede verse como un puente para relacionar ambos cabos del columpio, intentando evitar que la oscilación se convierta en un dilema. En este sentido, los criterios con los que contamos gracias a las ideas regulativas y a los juicios reflexionantes, conforman un puente para unir la sistematicidad con la adecuación empírica. El juicio empírico que resulte más armónico con esta unidad sistemática ideal, el juicio empírico que más se ajuste a ciertas virtudes epistémicas será nuestro mejor candidato a la verdad, será el juicio que más se conforme con nuestra realidad empírica, la cual, desde un inicio, es concebida como si se tratara de un sistema de fenómenos.

Por supuesto, estos criterios no vuelven necesarios nuestros juicios empíricos. Los juicios empíricos que consideramos verdaderos siempre pueden ser falsos pues son juicios contingentes. Y esto es así, en parte porque dichos juicios descansan, en última instancia, sobre un presupuesto inverificable: que la naturaleza es, de hecho, clasificable y sistematizable. Es este presupuesto lo que nos permite pensar que ciertas clasificaciones resultan más adecuadas que otras, pero no basta para hacer desaparecer todo el margen de indeterminación que existe cuando conceptualizamos la realidad. No obstante, esta contingencia de nuestro conocimiento empírico no representa una limitación de la filosofía kantiana, sino una virtud; siempre podemos cambiar y corregir no sólo juicios aislados sino partes enteras de nuestro sistema de conocimiento. Estrictamente hablando, la verdad empírica es finalmente inalcanzable, sólo podemos acercarnos asintóticamente a ella, permanece como un ideal regulativo, una pretensión constante que nos orienta siempre, pero la cual nunca podemos asegurar que se halla completamente satisfecha.

Para terminar, podríamos preguntarnos si Kant suscribiria esta manera nuestra de reordenar sus ideas acerca de la verdad. Quizá no fuera directamente su intención usar la doctrina del juicio reflexionante para solventar algunas de las dificultades de la verdad empírica de las que, no obstante, sí parece haber estado consciente. De cualquier manera, me parece que en su filosofía hay elementos suficientes para reordenar de la manera aquí sugerida su concepción de la verdad, aunque por supuesto no podamos atribuirle al sabio de Köninsberg esta labor de manera intencional y explícita. 
BiBLIOGRAFÍA

Guyer, Paul [1979], "The Theory of Reflective Judgment", cap. 2 de su libro Kant and the Claims of Taste, Harvard University Press, Cambridge, 1979.

_ [1990], "Reason and Reflective Judgment: Kant and the Significance of Systematicity", en Nous, no. 24, 1990, pp. 17-43.

_- [1996], "Los principios del juicio reflexionante", trad. Efraín Lazos, en este mismo volumen.

Kant, Immanuel [1781/1787], Crítica de la razón pura, trad. Pedro Ribas, Alfaguara, Madrid, 1988.

_-, [1800], Jäsche Logic, trad. al inglés e introd. Robert S. Hartman y Wolfgang Schwarz, Bobbs-Merrill Company Inc., Nueva York, 1974.

- [1790], Crítica de la facultad de juzgar, trad. e introd. Pablo Oyarzún, Monte Ávila Editores, Caracas, 1991. (Esta edición incluye la “'Primera Introducción' a la Crítica del juicio".)

Mc Dowell, John [1994], Mind and World, Harvard University Press, Cambridge, 1994.

Palacios, J. Miguel [1979], El idealismo trascendental. Teoría de la verdad, Gredos, Madrid, 1979.

Walker, Ralph, C.S. [1989], The Coherence Theory of Truth, Routledge, Londres, 1989 , caps. IV y VI. 